\title{
Resolución de Tareas Probabilísticas en Contexto Geométrico por Estudiantes de Educación Primaria
}

\author{
Luis Armando Hernández Solís' \\ Carmen Batanero" \\ María Magdalena Gea" \\ Rocío Álvarez-Arroyo" \\ 'Universidad Estatal a Distancia, San José - Costa Rica \\ "Universidad de Granada, Granada - España
}

RESUMEN - Resolución de Tareas Probabilísticas en Contexto Geométrico por Estudiantes de Educación Primaria ${ }^{1}$. Se presenta un estudio exploratorio de resolución de tareas probabilísticas de comparación de probabilidades y construcción del espacio muestral por parte de una muestra de 55 niños costarricenses de $6^{\circ}$ curso de primaria, analizando sus estrategias y errores. Comparando con investigaciones previas, se observa un mejor desempeño en el ítem en que se puede comparar casos favorables y posibles y en el que es necesaria la comparación de áreas, pero no hay diferencias en aquel en que se introduce como distractor el orden de colocación de los casos favorables. Se observa que, en general, se construye correctamente el espacio muestral en los casos de suceso posible y equiprobable, no así en los de sucesos imposible o seguro.

Palabras-Clave: Comparación de Probabilidades. Espacio Muestral. Contexto Geométrico. Educación Primaria.

ABSTRACT - Solving Probabilistic Tasks in Geometrical Context by Primary School Students. We present an exploratory study of solving probabilistic tasks proposed to a sample of 55 primary school $6^{\text {th }}$ grade Costa Rican children on comparison of probabilities and the construction of the sample space, analysing their strategies and errors. Comparing the results with previous investigations, an improvement is observed in the item in which the comparison of favorable and possible cases can be applied, and where the comparison of areas is necessary; however, there were no differences in the item in which the order in which the favorable cases are located is introduced as a distractor. The sample space is generally correctly built in the cases of possible and equiprobable event, but not in those of impossible or certain events.

Keywords: Comparison of Probability. Sample Space. Geometric Context. Primary Education.

Educação \& Realidade, Porto Alegre, v. 46, n. 3, e105401, 2021. 
Resolución de Tareas Probabilísticas en Contexto Geométrico por Estudiantes...

\section{Introducción}

Los conocimientos elementales de probabilidad son hoy día un requisito básico para el ciudadano, debido a las múltiples situaciones aleatorias a las que debe enfrentarse en su vida; también juegan un papel importante en el estudio posterior de la estadística (Batanero; Chernoff; Engel; Lee; Sánchez, 2016; Gal, 2005). Asimismo, tienen una participación importante en el desarrollo del pensamiento científico, donde muchos de sus procesos se describen mediante leyes aleatorias (Bryant; Nunes, 2012). Esta necesidad ha llevado a muchos países desarrollados a introducir la enseñanza de la probabilidad desde la educación primaria (por ejemplo, Australian Curriculum, Assessment and Reporting Authority, 2013; Common Core State Standards Initiative, 2010, Ministerio de Educación, Cultura y Deporte, 2014; National Council of Teachers of Mathematics, 2000).

En Costa Rica, se aprobaron nuevos programas escolares de Matemática en 2012, en los cuales el área de Estadística y Probabilidad adquirió un relieve mucho mayor respecto a los anteriores currículos nacionales, y una orientación más cercana a la naturaleza de esta disciplina. En estos programas se incluyen contenidos de probabilidad en toda la educación primaria (MEP, 2012); más concretamente, se espera que los niños y niñas alcancen las siguientes habilidades generales al finalizar cada ciclo educativo, comprendido por varios cursos:

Primer ciclo ( $\left(1^{\circ}\right.$ a $3^{\circ}$ cursos): Identificar situaciones aleatorias y seguras dentro de la cotidianidad y eventos asociados con ellas. Clasificar eventos aleatorios en más o menos probables para situaciones o experimentos particulares. Identificar eventos de acuerdo con los resultados simples que están vinculados con ellos (p. 147).

Segundo ciclo ( $4^{\circ}$ a $6^{\circ}$ cursos): Identificar eventos más probables, menos probables o igualmente probables de acuerdo con el número de resultados simples pertenecientes a cada evento. Determinar probabilidades elementales vinculadas con eventos particulares. Plantear y resolver problemas vinculados con situaciones aleatorias (p. 247).

Al incluir un nuevo contenido curricular, es importante asegurar que el estudiantado tenga las competencias necesarias para abordarlo con éxito, información que, generalmente, se puede adquirir de la investigación didáctica. Sin embargo, debido a la poca relevancia que se dio a los contenidos de probabilidad en currículos anteriores a 2012, ha habido una carencia de investigaciones referentes a la enseñanza y aprendizaje de la probabilidad en Costa Rica. Por otro lado, los estudios anteriores sobre el tema con niños y niñas de esta edad en otros países se han realizado cuando no habían recibido enseñanza en este tema.

Nuestra investigación trata de aportar información original sobre los estudiantes costarricenses que han iniciado estudios de probabilidad, que puede ser comparada con la de dichos estudios previos. Más concretamente, nos centramos en los niños y niñas de $6^{\circ}$ curso de educación primaria (11 y 12 años). Además, nos enfocamos en la probabi- 
lidad en el contexto geométrico, donde la investigación previa es más escasa.

La elección del nivel educativo apunta a dos razones: con este año se concluye la educación primaria; y segundo, este grupo de estudiantes había seguido los dos primeros cursos del segundo ciclo con el currículo actual de Matemática, ya que fue aprobado en 2012 y se aplicó de forma completa en todos los niveles educativos a partir del 2015. Es importante señalar que en el momento en que se desarrolló esta investigación, los participantes en la muestra apenas estaban iniciando el $6^{\circ}$ curso, por lo que aún no habían estudiado los contenidos de probabilidad correspondientes a dicho año escolar.

Específicamente, el objetivo de la investigación es explorar y describir las habilidades y razonamientos de una muestra de niños y niñas para resolver problemas de comparación de probabilidades simples en contexto geométrico, contrastando los resultados con la investigación previa, y evaluar sus destrezas en la construcción de espacios muestrales en dicho contexto.

\section{Marco Teórico}

El estudio de la capacidad de comparar probabilidades en experimentos aleatorios sencillos por parte de los niños comienza con Piaget e Inhelder (1951), quienes se apoyan en su teoría constructivista que asume que el aprendizaje surge de la experiencia, la actividad y el conocimiento previo. Según los autores, al intentar resolver un problema, el niño o niña utiliza los conocimientos que ya posee, y si no es capaz de resolverlo se le presenta un conflicto cognitivo que se soluciona mediante los procesos de asimilación y acomodación. La asimilación consiste en la incorporación (aceptación) por parte del sujeto de los datos o ideas nuevas, y la acomodación es el cambio o reestructuración de los ya existentes. Los autores sugieren que el conocimiento progresa en etapas de desarrollo, que tienen un orden establecido, aunque la edad en que un niño alcanza una de estas etapas puede variar.

Para estudiar la capacidad y razonamiento de los niños y niñas al comparar probabilidades sencillas, Piaget e Inhelder (1951) utilizaron fichas blancas marcadas o no con una cruz, introduciendo un pequeño número de fichas de cada tipo en urnas transparentes. Pidieron a los sujetos de su estudio elegir entre dos urnas de este tipo, preguntándoles cuál de ellas era preferible elegir, en un juego donde se ganaba al obtener una ficha marcada. Los autores fueron cambiando el número de fichas blancas (casos desfavorables) y marcadas (casos favorables) en las dos urnas y realizaron entrevistas mediante este juego a niños y niñas desde los 3 años y medio hasta los 13-14 años. Comparando las respuestas similares de grupos de sujetos de la misma edad, obtuvieron una descripción de tres estadios en el desarrollo de su razonamiento sobre este tipo de problemas.

El primer estadio (I) se divide en dos niveles. El nivel IA se caracteriza porque no se tienen los esquemas lógicos que permiten compren- 
Resolución de Tareas Probabilísticas en Contexto Geométrico por Estudiantes...

der la inclusión de la parte en un todo, la disyunción entre dos tipos de elementos y la conservación de las cantidades (por ejemplo, cuando se trasladan las fichas de lugar). Por ello, sólo se pueden resolver problemas donde existe doble imposibilidad (todas las fichas son blancas en las dos urnas), doble certeza (todas están marcadas) o certeza - imposibilidad (una urna con fichas blancas y otra con fichas marcadas). No se consideran todos los casos posibles y sólo se cuentan los casos favorables. En el nivel IB, los sujetos comparan solo un tipo de fichas (favorables o bien, desfavorables), y todavía no logran concebir los casos favorables como parte de los casos posibles (establecer una comparación de la parte con el todo); tampoco son capaces de comparar los casos favorables con los desfavorables (comparación parte-parte). Sin embargo, comienzan a comprender que la probabilidad depende del número de casos favorables.

El segundo estadio (II) comienza hacia los 7 años y también se divide en dos subniveles. En el nivel IIA, se pueden resolver problemas que implican una sola variable, es decir, cuando se necesita comparar solo los casos favorables o desfavorables. Se utiliza para ello operaciones aditivas (por ejemplo, restando el número de casos favorables a los desfavorables o viceversa, en cada urna y comparándolos). Se empieza a comprender la disyunción (cada caso es favorable o desfavorable), pero hay un fracaso sistemático en los casos en que la composición de casos favorables y desfavorables en las dos urnas es proporcional, pues no se ha adquirido la idea de fracción o de proporción. En el nivel IIB, el niño o niña comienza a resolver por correspondencia el problema, cuando la composición de las urnas es proporcional (por ejemplo, dos casos favorables por cada desfavorable, en ambas urnas).

En el estadio III, el sujeto es capaz de resolver fácilmente el caso de proporcionalidad y es capaz de pensar una solución general si el número de casos favorables y desfavorables es pequeño y la razón entre ellos es sencilla (por ejemplo, doble, triple, etc.), solución que va generalizando con la edad.

El éxito en la comparación de probabilidades, en el caso general, supone el razonamiento proporcional, cuyas etapas de desarrollo han sido analizadas por varios autores (p. ej., Karplus; Peterson, 1970; Noelting, 1980a; 1980b) y que se resumen en Behr et al. (1992) y Ben-Chaim, Keret y Ilany (2012). El más relevante para nuestro trabajo es Noelting (1980a; 1980b), quien a partir de un problema de comparación de dos mezclas (agua y zumo de naranja) subdivide las etapas de Piaget e Inhelder (1951), y determina las edades aproximadas en que se alcanzan, que serían para los niveles IA: 4 años, IB: 7 años, IIA: 8 años, IIB: 11 años y III: 12-13 años.

Finalmente, en nuestro trabajo se tiene en cuenta también la comprensión del espacio muestral (conjunto de sucesos posibles en un experimento). Dicha comprensión es un prerrequisito para que el niño o niña pueda realizar la comparación de probabilidades, ya que este problema requiere pensar en el conjunto de casos favorables y desfavorables como un todo del conjunto de casos posibles (Bryant; Nunes, 
2012). La estimación o comparación de probabilidades comienza por enumerar, o bien imaginar, el conjunto de elementos del espacio muestral, cuya determinación correcta es una parte esencial para resolver el problema (Chernoff, 2009). Sin embargo, son pocas las investigaciones centradas en la construcción del espacio muestral de un experimento simple por parte de los niños. Una excepción es el trabajo de Abrahamson (2006), en que pide a los niños y niñas escribir todas las posibilidades de un experimento que consiste en obtener cuatro bolas coloreadas de un conjunto de bolas de dos colores, lo que corresponde a un experimento compuesto, tarea en la que gran parte del estudiantado tiene dificultades. En este artículo, se trata una experiencia más sencilla, pues se trabaja solo con experimentos simples y en un mismo contexto.

\section{Antecedentes del Trabajo}

La investigación de Piaget e Inhelder inspiró una serie de trabajos sobre el razonamiento probabilístico de los niños, que se describen con detalle en Bryant y Nunes (2012), Jones, Langrall y Mooney (2007) y Langrall y Mooney (2005). A continuación, se resumen los más relacionados con nuestro trabajo.

Falk, Falk y Levin (1980) propusieron a niños y niñas de entre 4 y 11 años comparar probabilidades variando el número de casos favorables y posibles, y utilizando dos contextos: urnas con bolas y ruletas. En el caso de las ruletas, utilizaron sectores de dos colores con diferente número de sectores de cada color. A partir de los 6 años, los sujetos de su muestra presentaron algunas ideas correctas cuando el problema era sencillo. Los autores observaron que los niños y niñas no siempre usaron la misma estrategia para comparar probabilidades, sino que la estrategia dependía de los valores asignados a casos favorables y posibles. En el caso de las ruletas, muchos niños y niñas comparaban el número de sectores, en vez de utilizar las áreas. Por último, algunos sujetos tenían ideas irrelevantes (como el color favorito) que utilizaban en lugar de usar los datos para comparar probabilidades. Como indica Pratt (2000), las estrategias utilizadas para realizar juicios o comparaciones probabilísticas con frecuencia están sujetas a sesgos sistemáticos.

Truran (1994) realizó una investigación con 32 niños de 8 a 15 años, sobre comparación de probabilidades en urnas. Sus resultados identifican nuevas estrategias que amplían las descritas en la investigación de Piaget e Inhelder, como la descripción del contenido de la urna sin hacer una elección, dar una respuesta acertada sin justificación, usar estrategias diferentes para estimar la probabilidad en cada urna, preferencia por el número menor del total de bolas, comparación con proporciones sencillas conocidas y comparación entre razones de posibilidades a favor y en contra.

El estudio de mayor relevancia en el tema fue el realizado por Green (1983), quien realizó una evaluación del razonamiento probabilístico en estudiantes ingleses de 11 a 16 años con un test que reproducía en papel y lápiz los experimentos de Piaget e Inhelder. Algunos de 
$\underline{\text { Resolución de Tareas Probabilísticas en Contexto Geométrico por Estudiantes... }}$

sus ítems planteaban la comparación de probabilidades en contextos de urnas y ruletas. Las estrategias que encontraron en la comparación de probabilidades en urnas fueron: a) escoger la urna con mayor número de casos posibles; b) escoger la de mayor número de casos favorables; c) elegir la mayor diferencia entre casos favorables y desfavorables; d) elegir la mayor proporción entre casos favorables y desfavorables.

En contexto de ruletas, Green identifica los siguientes tipos de estrategias: a) comparar las áreas de las partes en que se divide la ruleta; b) analizar el número de porciones favorables o desfavorables, independientemente del área; c) comparaciones del número de sectores favorables o desfavorables, o ambos; d) utilizar razones de casos favorables o desfavorables; e) otras, como considerar la separación o continuidad de casos favorables o desfavorables.

Cañizares (1997) realizó un estudio con 320 niños españoles de 10 a 14 años y, entre otros problemas, propone los de comparación de probabilidades en contexto de urnas y ruletas. La autora describe las estrategias utilizadas por el estudiantado, que clasificaron en una y dos variables. Las estrategias de una variable consistían en comparar solo casos favorables, desfavorables o posibles; y las de dos variables en comparar de forma aditiva o multiplicativa los casos favorables y posibles. Cañizares deduce que el nivel más frecuente de razonamiento de los niños y niñas de su muestra es el IB a IIB, según categorización de Noelting (1980a; 1980b), siendo pocos sujetos los que alcanzan el IIIB. Algunas variables que influyen en la respuesta son la composición de las urnas (número de casos favorables y posibles) y la existencia de posibles sesgos en el contexto (por ejemplo, creencias de número favorito, equiprobabilidad o control de la situación).

En su trabajo, Green (1983) también evaluó la comprensión del lenguaje de la probabilidad en su muestra, incluyendo las expresiones imposible, posible, e igual posibilidad. En niños de $6^{\circ}$ curso obtuvo un $84 \%$ de respuestas correctas en el significado de imposible, $73 \%$ en posible y $18 \%$ en igual posibilidad. Cañizares (1997) por su parte, con las mismas tareas y también en niños y niñas de $6^{\circ}$ curso, obtuvo un $81,3 \%$ de respuestas correctas en el significado de imposible, $68,1 \%$ en posible y 42,9\% en igual posibilidad. Estos resultados apoyan la afirmación de Pratt (1998) que indica que la probabilidad es posiblemente la rama de las matemáticas que mayor distancia presenta entre la aplicación cotidiana y la comprensión formal de los conceptos y que, de hecho, el discurso matemático en el tema es, con frecuencia, diferente al lenguaje cotidiano.

En este trabajo se proponen tres ítems de comparación de probabilidades en ruletas (adaptación de los ítems 3 y 19 de Green, 1983) y trompos (adaptación del ítem 17 de Green, 1983), que fueron usados por Cañizares (1997) y otro relacionado con las ideas de suceso seguro, posible, equiprobable e imposible a una muestra de niños costarricenses que, a diferencia de los estudios citados, habían recibido instrucción en probabilidad a lo largo de la educación primaria. 


\section{Metodología}

La muestra utilizada estuvo formada por 55 niños y niñas de $6^{\circ}$ curso de educación primaria, 40 estudiantes de 11 años y 15 de 12 años, de los cuales 29 realizaban sus estudios en una institución privada y 26 en una institución pública (estatal-gratuita) de la provincia de Cartago, Costa Rica. Aunque las instituciones están situadas a menos de dos kilómetros de distancia, los estudiantes de la escuela privada provienen de diferentes cantones y distritos de la provincia, mientras el $90 \%$ de los estudiantes de la escuela pública viven en distrito donde está ubicada.

Ambas instituciones siguen el programa de estudios de Matemática del Ministerio de Educación Pública de Costa Rica - MEP (2012). Aunque, teóricamente, en la escuela pública se imparten 8 lecciones semanales de Matemática, y en la privada se imparten 5 (una de ellas dedicada específicamente a Estadística y Probabilidad), la cantidad de lecciones anuales en una institución pública es mucho menor que en la privada, debido a diferentes causas (actividades extracurriculares, capacitación, etc.).

De las entrevistas con las docentes a cargo de la asignatura de Matemática, se conoce que los niños y niñas estudiaron probabilidad de acuerdo a los programas de estudio MEP (2012) a partir del año 2016. Las consultas realizadas a los maestros indican que el tratamiento del tema se basó en el libro de texto, no habiendo evidencias de actividades realizando experimentos. En la revisión de los textos utilizados, sólo encontramos un ejercicio en contexto de ruletas, pero sin comparar probabilidades.

A dicha muestra se aplicó un cuestionario con cuatro ítems, todos ellos relacionados con la probabilidad en un contexto geométrico. Los tres primeros fueron tomados de la investigación de Cañizares (1997), quien los tradujo de otros similares de Green (1983), en los que se pide comparar probabilidades de un cierto suceso con dos ruletas o dos trompos y justificar la respuesta.

Las ruletas mostradas en el ítem 1 (Figura 1) se dividen en partes iguales, con cuatro y tres números, respectivamente, que corresponden a áreas equiprobables. El número de casos favorables (obtener 1) es el mismo en las dos ruletas, mientras que el de desfavorables es menor en la ruleta azul (2) que en la roja (3), por lo que la probabilidad de obtener 1 es de $1 / 3$ y $1 / 4$, respectivamente. Los niños y niñas podrían comparar los casos desfavorables en las dos ruletas para dar la solución correcta, sin recurrir a fracciones, lo que sería una estrategia del nivel de razonamiento IIA, según Piaget e Inhelder (1951). 
Resolución de Tareas Probabilísticas en Contexto Geométrico por Estudiantes...

\section{Figura 1 - Enunciado del ítem 1}

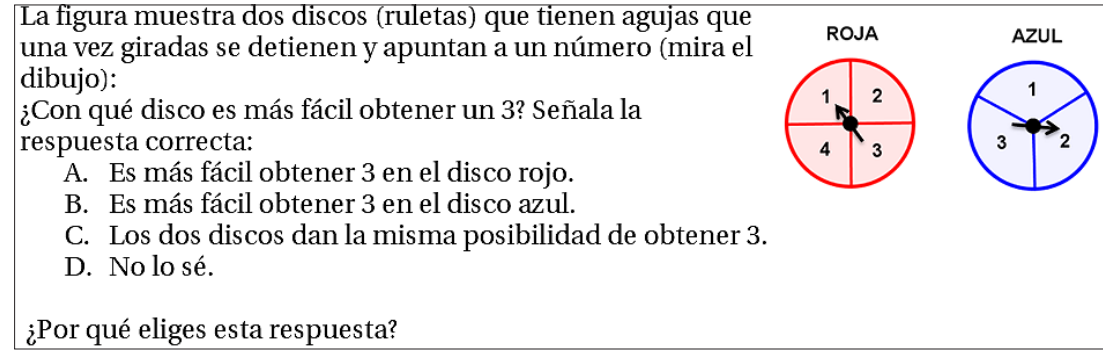

Fuente: Adaptado de Green (1983).

En el ítem 2 (Figura 2), se muestran dos trompos con la parte superior en forma de hexágono regular y dividida en seis triángulos iguales (misma área) en cada uno de los trompos, tres de los cuales corresponde al número 1 (casos desfavorables) y otros tres al número 2 (casos favorables). Por tanto, la probabilidad de obtener un 2 es la misma en ambos. Se podría resolver la tarea estableciendo una correspondencia entre los casos favorables y desfavorables, que es típica del nivel de razonamiento IIB, en la teoría de Piaget e Inhelder (1951). Por otro lado, el orden en que están situados los triángulos numerados con 1 y 2 en cada trompo es diferente: en el trompo amarillo se alternan los números, mientras que en el rojo son consecutivos. Esta colocación es un distractor que puede afectar al sujeto al comparar las probabilidades (Maury, 1984).

Figura 2 - Enunciado del ítem 2

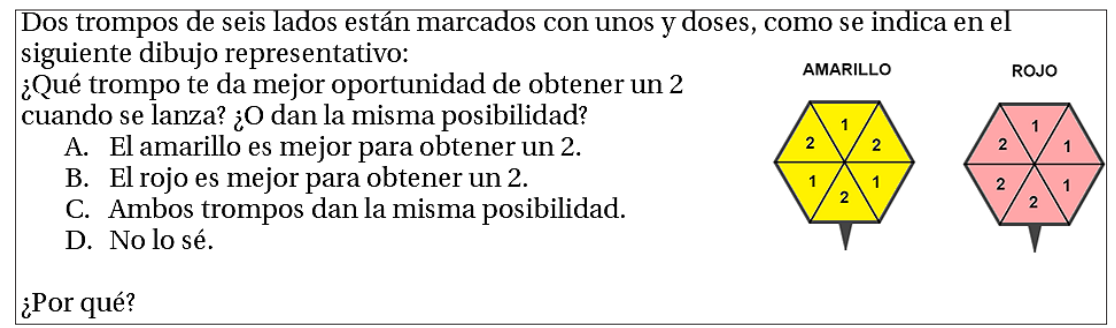

Fuente: Adaptado de Green (1983).

En el ítem 3 (Figura 3) se presentan dos ruletas con 6 sectores de diferente amplitud. En la ruleta marrón hay cuatro sectores numerados con el número 1 (casos favorables) y con el número 2 (casos desfavorables). La cantidad de casos favorables es mayor en la ruleta marrón que en la ruleta naranja (4 vs. 2), pero la amplitud y superficie ocupada por los casos favorables es mayor en esta última. Si se compara la razón entre casos favorables y desfavorables, se elegiría la ruleta marrón, que sería una respuesta incorrecta, pues la naranja tiene mayor superficie favorable al número 1. 


\section{Figura 3 - Enunciado del ítem 3}

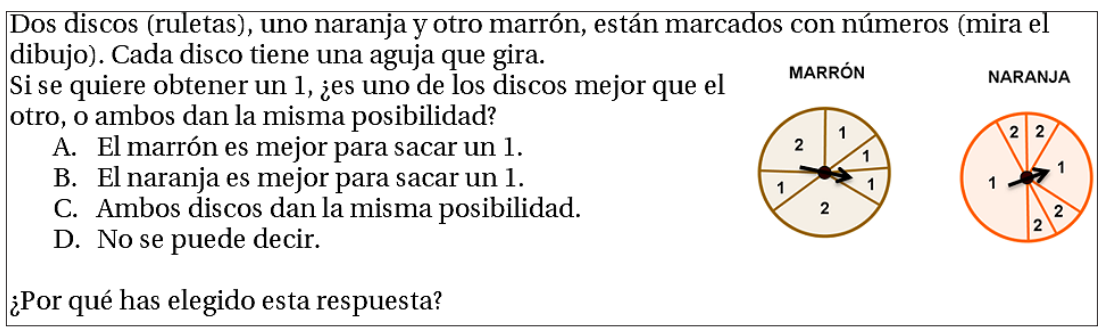

Fuente: Adaptado de Green (1983).

Se ha incluido un cuarto ítem (Figura 4) de elaboración propia, donde se pide crear un espacio muestral tal que, en un juego hipotético, el suceso María gana el juego (si obtiene el número 1) sea seguro, posible, equiprobable o imposible. La finalidad es evaluar la comprensión que revelan los sujetos de la muestra frente a este tipo de sucesos.

\section{Figura 4 - Enunciado del ítem 4}

María y Esteban juegan con una ruleta. María gana un confite si la aguja que gira cae en el 1 y Esteban gana un confite si cae en el 2. Coloca en las siguientes ruletas los números que consideres oportunos para que se cumpla:

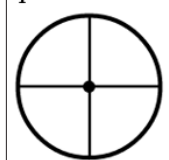

(A) María gana

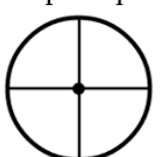

(B) Es posible que María gane

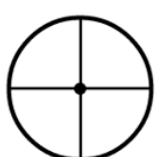

(C) María y Esteban tienen igual posibilidad de ganar

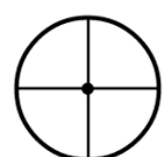

(D) Es imposible que María gane

Fuente: Elaboración propia.

El generador aleatorio utilizado en el juego descrito en el ítem 4 es una ruleta dividida en 4 partes iguales, por lo que el niño o niña puede crear un espacio muestral de hasta 4 sucesos diferentes (4 números distintos), pero si alguno de los números se repite (p. ej., construir una ruleta numerada $1,1,2,3$ ), se consigue un espacio muestral donde el suceso 1 tiene doble probabilidad que los restantes, y, por tanto, María tendría doble probabilidad de ganar que Esteban. Para conseguir el suceso seguro se debe repetir cuatro veces el número 1 (para que María gane siempre), mientras que para lograr el suceso imposible se debe excluir el número 1. En resumen, la resolución de la tarea requiere la intuición del experimento aleatorio y su resultado, así como de los tipos de sucesos.

\section{Resultados}

\section{Comparación de Probabilidades Geométricas}

En primer lugar, se analizó la corrección de la respuesta en cada uno de los tres primeros ítems para comparar los resultados con los obtenidos en las investigaciones previas. 
$\underline{\text { Resolución de Tareas Probabilísticas en Contexto Geométrico por Estudiantes... }}$

En la Tabla 1 se presentan las elecciones de respuesta al ítem 1, donde la mayoría de la muestra elije la opción correcta, con un porcentaje (80\%) algo más elevado que el obtenido en estudiantes de igual edad por Green (1983), 71\%, y Cañizares (1997), 79,1\%. Por tanto, puede decirse que este ítem ha resultado muy sencillo para los niños y niñas, que muestran el nivel de razonamiento IIA, según la clasificación de Piaget e Inhelder (1951). Se atribuye el mejor resultado en la presente investigación a la enseñanza recibida por los sujetos durante la educación primaria.

\section{Tabla 1 - Frecuencia y porcentaje de respuestas al ítem 1}

\begin{tabular}{lcc}
\hline Respuesta elegida & Frecuencia & Porcentaje \\
\hline Es más fácil obtener 3 en el disco rojo & 4 & 7,3 \\
Es más fácil obtener 3 en el disco azul (correcta) & 44 & 80,0 \\
Los dos discos dan la misma posibilidad de obtener 3 & 7 & 12,7 \\
\hline
\end{tabular}

Fuente: Elaboración propia.

En la Tabla 2 se presentan los resultados del segundo ítem, donde se observa más dificultad, aunque todavía el 50,9 \% de los niños y niñas responde correctamente.

Tabla 2 - Frecuencia y porcentaje de respuestas al ítem 2

\begin{tabular}{lcc}
\hline Respuesta elegida & Frecuencia & Porcentaje \\
\hline El amarillo es mejor para obtener un 2 & 12 & 21,8 \\
El rojo es mejor para obtener un 2 & 14 & 25,5 \\
Ambos trompos dan la misma posibilidad (correcta) & 28 & 50,9 \\
No sabe & 1 & 1,8 \\
\hline
\end{tabular}

Fuente: Elaboración propia.

En este ítem los resultados son similares a los de Green (49\%) y Cañizares (51,6\%), con estudiantes de igual edad. Por consiguiente, el sesgo introducido por la colocación de los casos favorables en el trompo no parece haberse superado con la enseñanza. Además, según Piaget e Inhelder (1951), la comparación de probabilidades en sucesos equiprobables corresponde a un nivel de razonamiento superior (este ítem se ubica en el nivel IIB).

En la Tabla 3 se reproducen los resultados en el tercer ítem, donde no se puede aplicar la regla de Laplace o las comparaciones de casos favorables o posibles, pues las áreas de cada parte en que se divide la ruleta son diferentes. Aun así, una gran parte de la muestra consigue resolver correctamente el problema $(63,6 \%)$, mientras que en la investigación de Green sólo lo logra el $43 \%$ de los sujetos de igual edad y el $46,2 \%$ en Cañizares, lo que de nuevo pensamos puede explicarse por la enseñanza recibida, en nuestro estudio. 
Hernández Solís; Batanero; Gea; Álvarez-Arroyo

Tabla 3 - Frecuencia y porcentaje de respuestas al ítem 3

\begin{tabular}{lcc}
\hline Respuesta elegida & Frecuencia & Porcentaje \\
\hline El marrón es mejor para sacar un 1 & 15 & 27,3 \\
El naranja es mejor para sacar un 1 (correcta) & 35 & 63,6 \\
Ambos discos dan la misma posibilidad & 5 & 9,1 \\
\hline \multicolumn{2}{c}{ Fuente: Elaboración propia }
\end{tabular}

Se observa en la Tabla 4 un mejor rendimiento, en general, en la escuela privada, pues comparando estos resultados separados con los de Green (1983) y Cañizares (1997), en los dos primeros ítems, la escuela pública tiene resultados inferiores a estos, mientras que la escuela privada se mantiene por encima y aumenta su diferencia. En el ítem tres, ambas escuelas se encuentran por encima de los porcentajes de las investigaciones previas.

Tabla 4-Porcentaje de respuestas correctas en los ítems 1, 2 y 3, según tipo de escuela, respecto a los obtenidos en investigaciones previas

\begin{tabular}{|c|c|c|c|c|}
\hline \multirow{2}{*}{ Ítem $\mathrm{N}^{\mathrm{o}}$} & \multicolumn{2}{|c|}{ Escuela } & \multirow{2}{*}{$\begin{array}{c}\text { Cañizares } \\
\text { (1997) }\end{array}$} & \multirow[t]{2}{*}{ Green (1983) } \\
\hline & Pública & Privada & & \\
\hline 1 & 65,4 & 93,1 & 79,1 & 71,0 \\
\hline 2 & 34,6 & 65,5 & 51,6 & 49,0 \\
\hline 3 & 57,7 & 69,0 & 46,2 & 43,0 \\
\hline
\end{tabular}

Fuente: Elaboración propia.

\section{Argumentos para Justificar la Comparación de Probabilidades}

Para comprender mejor las razones que llevaron a los sujetos de la muestra a elegir sus respuestas, se analizaron los argumentos aportados. Dichos argumentos fueron clasificados, mediante un análisis cualitativo, de acuerdo a las categorías descritas por Cañizares (1997) y Cañizares y Batanero (1997), que son las siguientes:

1. Comparación del número de casos favorables o posibles. Esta estrategia corresponde, según Piaget e Inhelder (1951), a utilizar una de las variables de la tarea y es propia del nivel de desarrollo IIA. En ella se compara el número de porciones numeradas en las dos ruletas que son favorables al caso pedido o bien el número total de porciones de la ruleta. No se usa o al menos no se hace referencia a los casos desfavorables, explícitamente, y funciona en el ítem 2 (porque el número de casos desfavorables es el mismo y todas las porciones tienen igual área) y en el ítem 1 (ya que las ruletas están dividas en partes iguales, con el mismo número de casos favorables aunque distinto de casos posibles); pero no en el ítem 3, pues no se tendría en cuenta la amplitud de cada sector de la ruleta. Algunos ejemplos son los siguientes:

E1: Porque tiene menos números y el azul no tiene el cuatro (respuesta B, ítem 1).

E2: Porque en las dos hay tres doses (respuesta C, ítem 2).

E3: Tiene más unos (respuesta A, ítem 3). 
$\underline{\text { Resolución de Tareas Probabilísticas en Contexto Geométrico por Estudiantes... }}$

2. Comparación explícita del número de casos desfavorables. Cuando explícitamente se cuentan los casos desfavorables y se comparan entre sí para justificar la respuesta, eligiendo la ruleta con menor número de casos desfavorables. También corresponde a una estrategia de una variable, pero más avanzada que comparar casos favorables o posibles. Es una estrategia que da una solución correcta cuando el número de casos favorables es igual en ambas ruletas. Es más elaborada que la anterior porque se tiene en cuenta el complementario del suceso pedido, lo que supone comprender la idea de disyunción. Algunos ejemplos se reproducen a continuación:

E4: Porque en el disco azul tienes 2 oportunidades de perder y en el disco rojo tienes 3 (respuesta $\mathrm{B}$, ítem 1 ).

E5: Porque en el azul no está el 4 (respuesta B, ítem 1).

3. Comparación explícita del número de casos favorables y desfavorables. Es una estrategia de dos variables, según Cañizares y Batanero (1997), donde se utilizan todos los datos del enunciado. Se comparan los casos favorables y desfavorables en forma aditiva, como muestra E6, o se realiza comparación multiplicativa como muestra E7. Piaget e Inhelder (1951) advierten que estas estrategias no permiten, en general, resolver cualquier problema (como sucede en el ítem 3, que lleva a error porque no se tiene en cuenta el área de cada sector), aunque pueden llevar a éxito dependiendo de los datos (como ocurre en el ítem 2).

E6: Porque en la naranja hay más 1 que 2 (respuesta B, ítem 3).

E7: Tienen 3 veces 2 y 3 veces 1 (respuesta C, ítem 2).

4. Comparación del área ocupada por el número pedido. Esta es una estrategia que sólo puede aplicarse en un contexto de probabilidad geométrica y puede utilizarse con éxito en todos los ítems propuestos. En este trabajo se utiliza en el ítem 3, donde la comparación de casos favorables o desfavorables no funciona y el estudiante debiera recurrir a comparar el área total cubierta (amplitud de las superficies), como por ejemplo:

E8: Porque el 1 tiene más espacio y es probable que salga el 1 (respuesta B, ítem 3).

E9: Porque el uno tiene el espacio más grande (respuesta B, ítem 3).

5. Compara áreas y número de casos desfavorables o favorables. Es una combinación de las dos estrategias anteriores, lo que supone mayor nivel de razonamiento, como por ejemplo:

E10: Yo elegí esa respuesta porque hay menos números y los espacios son más grandes (repuesta B, ítem 3 ).

E11: Porque hay menos números, por lo que el 3 tiene un espacio más grande (repuesta $B$, ítem 1 ).

6. Sesgo de equiprobabilidad. Algunos niños o niñas hacen referencia al azar (expresado también como suerte) para deducir que cualquier suceso es igualmente posible, sin tener en cuenta el área ocupada ni el número de casos favorables o posibles. Se muestra en estas res- 
puestas (E12) un ejemplo del sesgo de equiprobabilidad, descrito por Lecoutre (1992), consistente en equiparar aleatoriedad y equiprobabilidad, que también apareció en Cañizares (1997).

E12: Solo hay diferencia de un número el 4 pero pueden tener una misma probabilidad (respuesta C, ítem 1).

7. Consideraciones físicas. Se han encontrado puntualmente otros argumentos basados en la colocación de los números en el trompo, la fuerza que se da a la aguja o consideraciones similares. En los ejemplos E13 y E17 se utiliza la creencia subjetiva de que el orden de colocación de los números en la ruleta influye en la probabilidad, incluso cuando las áreas correspondientes a los casos favorables y posibles sean la misma (caso E13). Hay respuestas que revelan una preferencia por el posible resultado, según ubicación (en las esquinas, E14), una dependencia de la posición inicial de la aguja (E15) o incluso de la fuerza que se le imprime a la ruleta o trompo (E16, E18).

E13: El amarillo, porque está más distribuido (respuesta A, ítem 2).

E14: Porque seguramente cae en las esquinas (respuesta A, ítem 2).

E15: Porque en el disco azul la aguja apunta al dos, y el siguiente número será el tres, en cambio en el disco rojo apunta al uno y el siguiente será el dos (respuesta B, ítem 3).

E16: Porque en esto todo depende de la fuerza (respuesta C, ítem 1).

E17: Porque los dos están cerca del 1 (respuesta C, ítem 3).

E18: Si la giras con cuidado si va salir la 3 (respuesta A, ítem 1).

En la Tabla 5 se presentan los resultados referentes a los argumentos identificados para cada ítem, siendo en los dos primeros ítems correctas las estrategias de comparación de casos favorables, desfavorables o posibles (el número de casos favorables es igual) junto a la comparación de áreas, que solo funciona en el tercer ítem.

En el ítem 1 predomina la comparación de los casos posibles, que enmascara la comparación de casos desfavorables al haber uno solo favorable. Así, implícitamente se utiliza la disyunción y se concibe el espacio muestral como unión de casos favorables y desfavorables. Es decir, si el sujeto indica que en una ruleta hay más posibilidad porque hay menos números, se refiere implícitamente al número de casos desfavorables. En la investigación de Green (1983) solo el 28\% de los niños y niñas de la misma edad que los que participan en el presente estudio usaron este argumento y el 30,8\% en la de Cañizares (1997). Por tanto, los resultados en nuestra muestra superan los de dichos estudios. 
$\underline{\text { Resolución de Tareas Probabilísticas en Contexto Geométrico por Estudiantes... }}$

Tabla 5 - Frecuencia y porcentaje de estrategias en los ítems

\begin{tabular}{|c|c|c|c|c|c|c|}
\hline \multirow{2}{*}{ Estrategia } & \multicolumn{2}{|c|}{ Ítem 1} & \multicolumn{2}{|c|}{ Ítem 2} & \multicolumn{2}{|c|}{ Ítem 3} \\
\hline & $\mathrm{N}$ & $\%$ & $\mathrm{~N}$ & $\%$ & $\mathrm{~N}$ & $\%$ \\
\hline 1. Comparar casos favorables o posibles & 27 & $49,1^{*}$ & 7 & $12,7^{*}$ & 13 & 23,6 \\
\hline 2. Comparar casos desfavorables & 5 & $9,1^{*}$ & & & & \\
\hline 3. Comparar casos favorables y desfavorables & & & 14 & $25,5^{*}$ & 1 & 1,8 \\
\hline 4. Comparar áreas & 6 & $10,9^{*}$ & & & 27 & $49,3^{*}$ \\
\hline 5. Áreas y casos favorables o desfavorables & 5 & $9,1^{*}$ & & & 4 & $7,3^{*}$ \\
\hline 6. Sesgo de equiprobabilidad & 6 & 10,9 & 3 & 5,5 & 2 & 3,6 \\
\hline 7. Consideraciones físicas & 6 & 10,9 & 30 & 54,5 & 7 & 12,7 \\
\hline No sabe & & & 1 & 1,8 & 1 & 1,8 \\
\hline
\end{tabular}

En el ítem 2 son correctas tanto la comparación de casos posibles o favorables, como la de casos favorables y desfavorables, y entre los dos se acerca al 40\% de uso. En el caso de Green, el 35\% de los sujetos utiliza la comparación de casos favorables y posibles y la situación de los casos favorables y desfavorables, y en la investigación de Cañizares, alrededor del $25 \%$, por lo que, de nuevo los resultados del estudio actual son mejores. Sin embargo, en este estudio aparece un porcentaje muy alto de niños y niñas que utilizan consideraciones físicas irrelevantes (54,5\%), mientras que en el trabajo de Cañizares supone solo el $21 \%$, siendo en su caso la referencia a la suerte o equiprobabilidad algo mayor que en este caso.

En el tercer ítem predomina la comparación de áreas, a veces combinada con comparación de casos favorables o desfavorables. La proporción de argumentos correctos presentados en este artículo es mayor que en Green y en Cañizares con niños y niñas de igual edad (41\% y 41,8\% respectivamente), mientras que la comparación de casos favorables y desfavorables (estrategia incorrecta) es menor que en estos autores.

De acuerdo al tipo de escuela (Tabla 6), en general los porcentajes fueron similares excepto en el ítem 2, donde el $41,4 \%$ de los y las estudiantes de la escuela privada optó por la estrategia 3 , frente al $7,7 \%$ de los y las estudiantes de la escuela pública. Es importante señalarlo, ya que de acuerdo a Cañizares y Batanero (1997), esta estrategia es más avanzada al considerar dos variables. Este ítem, en general, fue más complejo para los niños de la escuela pública, donde menos del $27 \%$ tuvo argumentos correctos, y el argumento basado en consideraciones físicas tiene un índice del $65,4 \%$. 
Hernández Solís; Batanero; Gea; Álvarez-Arroyo

Tabla 6 - Porcentaje de estrategias en los ítems 1 a 3 por tipo de escuela

\begin{tabular}{|c|c|c|c|c|c|c|}
\hline \multirow{2}{*}{ Estrategia } & \multicolumn{2}{|c|}{ Ítem 1} & \multicolumn{2}{|c|}{ Ítem 2} & \multicolumn{2}{|c|}{ Ítem 3} \\
\hline & Priv. & Púb. & Priv. & Púb. & Priv. & Púb. \\
\hline 1. Comparar casos favorables o posibles & $55,2^{*}$ & $42,3^{*}$ & $10,3^{*}$ & $19,2^{*}$ & 17,2 & 30,8 \\
\hline 2. Comparar casos desfavorables & $10,3^{*}$ & $7,7^{*}$ & & & & \\
\hline 3. Comparar casos favorables y desfavorables & & & $41,4^{*}$ & $7,7^{*}$ & 3,4 & \\
\hline 4. Comparar áreas & $10,3^{*}$ & $11,5^{*}$ & & & $55,2^{*}$ & $46,2^{*}$ \\
\hline 5. Áreas y casos favorables o desfavorables & $10,3^{*}$ & $7,7^{*}$ & & & $6,9^{*}$ & $3,8^{*}$ \\
\hline 6. Sesgo de equiprobabilidad & 6,9 & 15,4 & & 3,8 & 3,4 & 3,8 \\
\hline 7. Consideraciones físicas & 6,9 & 15,4 & 48,3 & 65,4 & 10,3 & 15,4 \\
\hline No sabe & & & & 3,8 & 3,4 & \\
\hline
\end{tabular}

\section{Construcción del Espacio Muestral}

En la Tabla 7 se presenta la frecuencia y porcentaje de estudiantes según el tipo de espacio muestral que construyen, ya sea un suceso seguro, muy probable (probabilidad igual o superior a 0,75 ), posible (probabilidad entre 0,25 y 0,75 ), equiprobable, poco probable (probabilidad igual o inferior a 0,25 ) e imposible, en función de lo pedido en cada apartado del ítem (que sea suceso seguro, posible, equiprobable o imposible).

Tabla 7 - Frecuencia y porcentaje de diferentes categorías de respuesta al ítem 4

\begin{tabular}{|c|c|c|c|c|c|c|c|c|}
\hline \multirow{3}{*}{$\begin{array}{l}\text { Espacio muestral construido } \\
\text { correspondiente a un... }\end{array}$} & \multicolumn{8}{|c|}{ Tipo de suceso pedido } \\
\hline & \multicolumn{2}{|c|}{ Seguro } & \multicolumn{2}{|c|}{ Posible } & \multicolumn{2}{|c|}{$\begin{array}{l}\text { Equipro- } \\
\text { bable }\end{array}$} & \multicolumn{2}{|c|}{ Imposible } \\
\hline & $\mathrm{N}$ & $\%$ & $\mathrm{~N}$ & $\%$ & $\mathrm{~N}$ & $\%$ & $\mathrm{~N}$ & $\%$ \\
\hline Suceso seguro & 19 & $34,5^{*}$ & & & & & 1 & 1,8 \\
\hline Suceso muy probable & 23 & 41,8 & 20 & $36,4^{*}$ & & & 10 & 18,2 \\
\hline Suceso equiprobable & 6 & 10,9 & 27 & $49,1^{*}$ & 50 & $90,9^{*}$ & 9 & 16,4 \\
\hline Suceso poco probable & 5 & 9,1 & 6 & $10,9^{*}$ & 3 & 5,5 & 19 & 34,5 \\
\hline Suceso imposible & & & & & & & 14 & $25,5^{*}$ \\
\hline En blanco & 2 & 3,6 & 2 & 3,6 & 2 & 3,6 & 2 & 3,6 \\
\hline
\end{tabular}

Son pocos los sujetos que no responden a los apartados, siendo lo más sencillo comprender la idea de suceso posible, correctamente respondida por la mayoría de la muestra $(96,4 \%)$, aunque casi la mitad construye un espacio muestral correspondiente al suceso equiprobable y esto puede estar asociado a la creencia de que en un suceso aleatorio todos los resultados son equiprobables, llamado sesgo de equiprobabilidad (Lecoutre, 1992), aunque en este caso sea válida. También resulta 
Resolución de Tareas Probabilísticas en Contexto Geométrico por Estudiantes...

sencillo identificar el suceso equiprobable, pues una amplia mayoría $(90,9 \%)$ responde correctamente. Respecto al suceso seguro, más de una tercera parte de la muestra lo construyó correctamente $(34,5 \%)$, pero también una alta frecuencia lo interpretó como muy probable $(41,8 \%)$. Puede observarse que la mayor dificultad estuvo en el suceso imposible, que generalmente fue considerado como poco probable $(34,5 \%)$ y solo una cuarta parte lo construyó correctamente.

Al comparar con los trabajos de Green (1983) y Cañizares (1997), los resultados actuales son mejores en la interpretación del suceso seguro (los autores obtienen un $16 \%$ y $26,4 \%$ de éxito, respectivamente) en los niños de $6^{\circ}$ curso, del suceso equiprobable ( $18 \%$ y $42,9 \%$, respectivamente) y del suceso posible ( $73 \%$ y $68,1 \%$, respectivamente). El único suceso donde los resultados del presente estudio han sido peores es en la identificación del suceso imposible, cuyos autores obtienen unas tasas de respuestas correctas del $84 \%$ y $81,3 \%$, respectivamente. Hay que matizar, no obstante, que la tarea planteada en el presente estudio es más difícil que las propuestas por los autores, quienes pidieron a los niños proponer sinónimos o escribir frases con los términos analizados, mientras que en esta investigación se les pide construir el espacio muestral de un posible experimento.

Respecto al tipo de escuela (Tabla 8), los resultados son muy similares, aunque cabe señalar que los participantes de la escuela pública tuvieron todas las respuestas correctas en el suceso equiprobable; y los de la escuela privada tuvieron un rendimiento levemente mejor respecto a los de la escuela pública en el suceso imposible.

\section{Tabla 8 - Frecuencia y porcentaje de diferentes categorías de respuesta al ítem 4 según tipo de escuela}

\begin{tabular}{|c|c|c|c|c|c|c|c|c|}
\hline \multirow{3}{*}{ Espacio muestral construido } & \multicolumn{8}{|c|}{ Tipo de suceso pedido } \\
\hline & \multicolumn{2}{|c|}{ Seguro } & \multicolumn{2}{|c|}{ Posible } & \multicolumn{2}{|c|}{$\begin{array}{l}\text { Equipro- } \\
\text { bable }\end{array}$} & \multicolumn{2}{|c|}{ Imposible } \\
\hline & Priv. & Púb. & Priv. & Púb. & Priv. & Púb. & Priv. & Púb. \\
\hline Corresponde al suceso seguro & $34,5^{*}$ & $34,6^{*}$ & & & & & & 3,8 \\
\hline $\begin{array}{l}\text { Corresponde a suceso muy } \\
\text { probable }\end{array}$ & 41,4 & 42,3 & $31,0^{*}$ & $42,3^{*}$ & & & 10,3 & 26,9 \\
\hline $\begin{array}{l}\text { Corresponde a suceso equipro- } \\
\text { bable }\end{array}$ & 6,9 & 15,4 & $51,7^{*}$ & $46,2^{*}$ & $82,8^{*}$ & $100,0^{*}$ & 13,8 & 19,2 \\
\hline $\begin{array}{l}\text { Corresponde a suceso poco } \\
\text { probable }\end{array}$ & 10,3 & 7,7 & $10,3^{*}$ & $11,5^{*}$ & 10,3 & & 37,9 & 30,8 \\
\hline $\begin{array}{l}\text { Corresponde al suceso impo- } \\
\text { sible }\end{array}$ & & & & & & & $31,0^{*}$ & $19,2^{*}$ \\
\hline No construye & 6,9 & & 6,9 & & 6,9 & & 6,9 & \\
\hline
\end{tabular}


Hernández Solís; Batanero; Gea; Álvarez-Arroyo

\section{Relación entre Comparación de Probabilidades y Construcción del Espacio Muestral}

En la Tabla 9 se presenta la distribución conjunta del número de respuestas correctas en las tareas de comparación de probabilidades (ítems 1 a 3) y construcción del espacio muestral, según diferentes tipos de suceso (ítem 4). Todos los porcentajes se calculan respecto al total de la fila, excepto los porcentajes del total de niños y niñas que obtienen 0 , 1, 2, 3 o 4 espacios muestrales correctos, que se calcula respecto al total de la muestra.

Únicamente 2 sujetos construyeron incorrectamente los cuatro espacios muestrales pedidos, siendo lo más frecuente obtener dos correctos $(56,4 \%)$ seguido de cuatro correctos $(21,8 \%)$ y tres correctos (14,5\%); solo 2 sujetos respondieron correctamente a una única construcción del espacio muestral solicitado. Por tanto, la construcción del espacio muestral fue una tarea compleja, pues poco más de un tercio de las respuestas tiene al menos tres espacios muestrales correctamente construidos. Se considera que ello es debido a la confusión general entre sucesos imposible y poco probable o entre sucesos seguro y muy probable. Por otro lado, solo dos sujetos fallan en todas las comparaciones de probabilidades, siendo lo más frecuente responder correctamente a dos de ellas $(36,4 \%)$ o a las tres $(30,9 \%)$.

Observamos también la relación entre el número de respuestas correctas en comparación de probabilidades y en la construcción del espacio muestral. Así, los 2 sujetos que fallan todas las preguntas de comparación de probabilidades solo llegan a completar adecuadamente dos espacios muestrales; y el porcentaje de los que construyen bien los cuatro espacios muestrales crece hasta el 35,3\% de los niños que resuelven correctamente todas las tareas de comparación de probabilidades.

Tabla 9 - Frecuencias y porcentajes del número de respuestas correctas en comparación de probabilidades y construcción del espacio muestral

\begin{tabular}{|c|c|c|c|c|c|c|c|c|}
\hline \multirow{2}{*}{\multicolumn{2}{|c|}{$\begin{array}{c}\mathrm{N}^{o} \text { comparación proba- } \\
\text { bilidades correctas }\end{array}$}} & \multicolumn{6}{|c|}{$\mathrm{N}^{\circ}$ de espacios muestrales correctos } & \multirow{3}{*}{$\frac{\% \text { Total }}{3,6}$} \\
\hline & & \multirow[t]{2}{*}{0} & \multirow[t]{2}{*}{1} & \multirow{2}{*}{$\frac{2}{2}$} & \multirow[t]{2}{*}{3} & \multirow[t]{2}{*}{4} & \multirow{2}{*}{$\frac{\text { Total }}{2}$} & \\
\hline 0 & Frecuencia & & & & & & & \\
\hline & $\%$ por fila & & & 100,0 & & & & \\
\hline 1 & Frecuencia & & 1 & 9 & 3 & 3 & 16 & 29,1 \\
\hline & $\%$ por fila & & 6,3 & 56,3 & 18,8 & 18,8 & & \\
\hline 2 & Frecuencia & 1 & 1 & 13 & 2 & 3 & 20 & 36,4 \\
\hline & $\%$ por fila & 5,0 & 5,0 & 65,0 & 10,0 & 15,0 & & \\
\hline 3 & Frecuencia & 1 & & 7 & 3 & 6 & 17 & 30,9 \\
\hline & $\%$ por fila & 5,9 & & 41,2 & 17,6 & 35,3 & & \\
\hline Total & Frecuencia & 2 & 2 & 31 & 8 & 12 & 55 & 100,0 \\
\hline 10tar & \% del total & 3,6 & 3,6 & 56,4 & 14,5 & 21,8 & 100,0 & 100,0 \\
\hline
\end{tabular}

Educação \& Realidade, Porto Alegre, v. 46, n. 3, e105401, 2021. 
Resolución de Tareas Probabilísticas en Contexto Geométrico por Estudiantes...

\section{Discusión y Conclusiones}

El análisis de las respuestas en la comparación de probabilidades en ruletas y trompos indica mejores resultados en el presente estudio que los obtenidos con niños y niñas de la misma edad en investigaciones previas, que fueron realizadas en un periodo en que no se impartía ningún tipo de enseñanza de la probabilidad elemental en las escuelas. Estas diferencias se encuentran principalmente en el primer ítem, que se puede resolver mediante comparación de casos favorables y desfavorables, y en el tercero, cuya solución se fundamenta en la comparación de áreas. Se atribuye este mejor razonamiento a la enseñanza recibida por los niños y niñas actualmente, que confirma las teorías de Fischbein (1975) sobre la importancia de aprovechar la base intuitiva con la probabilidad y proporcionar instrucción al respecto, apoyadas en lo posible en material manipulativo.

Sin embargo, la enseñanza no parece haber influido en las respuestas al segundo ítem, pues se obtienen resultados similares a los trabajos de Green (1983) y Cañizares (1997), donde la disposición de los números en el trompo se ha tenido en cuenta como un elemento subjetivo y distractor en la comparación de probabilidades. En este sentido, se considera necesario que el estudiantado realice experimentos con dispositivos físicos similares a los mostrados en los enunciados de los ítems para que poco a poco puedan corregir los sesgos de razonamiento inadecuados. De acuerdo a Pratt (2000), son muchos los materiales que pueden servir de recursos para apoyar la construcción de intuiciones correctas sobre el azar. Esta recomendación sigue el principio de que el conocimiento es construido activamente por el sujeto y no recibido pasivamente del entorno (Piaget; Inhelder, 1951); de ahí surge la importancia de una enseñanza activa también en el campo de la probabilidad.

En la tarea de construcción de espacios muestrales fue sencillo construir el espacio muestral asociado a un suceso posible o equiprobable, pero hubo muchos en el caso de los sucesos seguro o imposible, reforzando los resultados de investigaciones como las de Green (1983) y Cañizares (1997) que indican la dificultad que tienen los niños y niñas con el lenguaje de probabilidad.

La comprensión de este lenguaje y del espacio muestral asociado a experimentos sencillos se ha mostrado relacionada con la tarea de comparación de probabilidades en este trabajo, lo que indica la necesidad de reforzar la formación de los y las estudiantes en dichas tareas. De hecho, algunos investigadores también apoyan el trabajo con la probabilidad desde la primera infancia, para introducir a los niños y niñas en el lenguaje del azar. Así, Alsina (2012), por su parte, analiza las posibilidades de juegos que se practican habitualmente en la niñez (p. ej., con dados). En las conversaciones, juegos, cuentos y canciones infantiles se puede observar con frecuencia referencias al azar. Por ejemplo, los niños y niñas usan canciones para echar a suertes en el escondite o en el rescate, utilizan juegos de mesa con datos o ruletas. Estos juegos pueden utilizarse con fines educativos para reforzar la intuición proba- 
bilística de los niños y niñas y su diferenciación de los sucesos seguros, imposibles y posibles.

Es claro que una buena enseñanza de la probabilidad pasa por tener profesores entusiasmados por el tema y bien preparados. Sin embargo, algunos profesores y estudiantes para profesor de educación primaria pueden sentirse inseguros al enseñar la probabilidad, por no haber recibido suficiente formación sobre didáctica de la probabilidad o no tener experiencia en su enseñanza (Alpízar et al., 2012; Alpízar; Chavarría; Oviedo, 2015). Ello puede llevarles a reducir u omitir su enseñanza, alegando razones como la falta de tiempo debido a lo apretado de los programas o inseguridad para enseñarlo.

Una aportación del artículo es el material presentado, que incluye los ítems propuestos a los niños y niñas, el análisis detallado de las respuestas de los mismos y su clasificación. Todo ello puede servir para organizar cursos de formación del profesorado donde estos analicen los ítems y las tareas prototípicas de los niños y niñas en diferentes niveles de desarrollo cognitivo. Es importante que reflexionen sobre las demandas cognitivas de las tareas planteadas a los niños, sobre sus formas de razonamiento, los posibles sesgos y los mejores modos de superarlos y finalmente que propongan tareas similares que puedan desarrollar con sus estudiantes.

Recibido en 15 de julio de 2020 Aprobado en 10 de mayo de 2021

\section{Nota}

1 Agradecimientos-Proyecto PID2019-105601GB-I00 / AEI / 10.13039/501100011033 y Grupo de Investigación FQM-126 (Junta de Andalucía).

\section{Referencias}

ABRAHAMSON, Dor. The Shape of Things to Come: the computational pictograph as a bridge from combinatorial space to outcome distribution. International Journal of Computers for Mathematical Learning, Netherlands, v. 11, n. 1, p. 137-146, 2006.

ALPÍZAR, Marianela; BARRANTES, José Pablo.; BOLAÑOS, Helen; CÉSPEDES, Michael; DELGADO, Evelyn; FREER, Dylana; PADILLA, Erick; VÍQUEZ, María Fernanda. Aspectos relevantes sobre la formación docente en I y II ciclos en los temas Probabilidad y Estadística. Revista Electrónica Educare, Costa Rica, v. 16, n. 2, p. 113-129, 2012. Disponible en: <http://doi.org/10.15359/ree.16-2.7>. Aceso en: 02 jan. 2020.

ALPÍZAR, Marianela; CHAVARRÍA, Laura; OVIEDO, Katalina. Percepción de un grupo de docentes de I y II ciclo de educación general básica de escuelas públicas de Heredia sobre los temas de estadística y probabilidad. Actualidades Investigativas en Educación, Costa Rica, v. 15, n. 1, p. 1-23, 2015. Disponible en: $<$ dx.doi.org/10.15517/aie.v15il.17728>. Aceso en: 02 jan. 2020.

ALSINA, Ángel. La estadística y la probabilidad en Educación Infantil: conocimientos disciplinares, didácticos y experienciales. Revista de Didácticas Específicas, Madrid, v. 7, p. 4-22, 2012. 
$\underline{\text { Resolución de Tareas Probabilísticas en Contexto Geométrico por Estudiantes... }}$

AUSTRALIAN CURRICULUM, ASSESSMENT AND REPORTING AUTHORITY (ACARA). The Australian curriculum: Mathematics. Sidney, NSW: ACARA, 2013. BATANERO, Carmen; CHERNOFF, Egan; ENGEL, Joachim; LEE, Hollylynne; SÁNCHEZ, Ernesto. Research on teaching and learning probability. Hamburg: Springer, 2016.

BEHR, Merilyn; HAREL, Guershon; POST, Thomas; LESH, Richard. Rational number, ratio and proportion. In: GROUWS, Douglas (Ed.). Handbook of research in mathematics teaching and learning. New York: Macmillan, 1992. P. 296-333.

BEN-CHAIM, David; KERET, Yaffa; ILANY, Bat-Shevallany. Ratio and proportion: research and teaching in mathematics teachers' education. Rotterdam: Sense Publisher, 2012.

BRYANT, Peter; NUNES, Terezinha. Children's understanding of probability: a literature review (full report). London: Nuffield Foundation, 2012.

CAÑIZARES, María Jesús. Influencia del razonamiento proporcional y combinatorio y de creencias subjetivas en las intuiciones probabilísticas primarias. 1997. Tesis (Doctoral) - Universidad de Granada, Granada, 1997.

CAÑIZARES, María Jesús; BATANERO, Carmen. Influencia del razonamiento proporcional y de las creencias subjetivas en la comparación de probabilidades. UNO: Revista de didáctica de las matemáticas, España, v. 14, p. 99-114, 1997.

CHERNOFF, Egan. Sample space partitions: an investigative lens. Journal of Mathematical Behavior, United States, v. 28, p. 19-29, 2009.

COMMON CORE STATE STANDARDS INITIATIVE. Common Core State Standards for Mathematics. Washington, DC: National Governors Association Center for Best Practices and the Council of Chief State School Officers, 2010.

FALK, Ruma; FALK, Raphael; LEVIN, Iris. A potential for learning probability in young children. Educational Studies in Mathematics, Amsterdam, v. 11, p. 181-204, 1980.

FISCHBEIN, Efraim. The intuitive sources of probabilistic thinking in children. Dordrecht: Reidel, 1975.

GAL, Iddo. Towards 'probability literacy' for all citizens: building blocks and instructional dilemmas. In: JONES, Graham (Org.). Exploring probability in school: challenges for teaching and learning. New York: Springer, 2005. P. 39-63.

GREEN, David. A survey of probability concepts in 3000 pupils aged 11-16 years. In: GREY, David R.; HOLMES, Peter; BARNETT, Vic; CONSTABLE, Gillian M. Proceedings of the First International Conference on Teaching Statistics. Sheffield, UK: Teaching Statistics, Trust, 1983. P. 766-783.

JONES, Graham; LANGRALL, Cynthia; MOONEY, Edward. Research in probability: responding to classroom realities. In: LESTER, Frank (Ed.). Second handbook of research on mathematics teaching and learning. Greenwich, CT: Information Age Publishing y NCTM, 2007. P. 909-955.

KARPLUS, Robert; PETERSON, Rita. Intellectual development beyond elementary school II: ratio, a survey. School Science and Mathematics, Indiana, v. 70, p. 813-120, 1970.

LANGRALL, Cynthia; MOONEY, Edward. Characteristics of elementary school students' probabilistic thinking. In: JONES, Graham (Ed.). Exploring probability in school: challenges for teaching and learning. Dordrech, Netherlands: Kluwer, 2005. P. 95-119. 
LECOUTRE, Marie. Cognitive models and problem spaces in 'purely random' situations. Educational Studies in Mathematics, New York, v. 23, p. 557-568, 1992. MAURY, Sylvette. La quantification des probabilités: analyse des arguments utilisés par les élèves de classe de seconde. Recherches en Didactique des Mathématiques, France, v. 5, n. 2, p. 187- 214, 1984.

MINISTERIO DE EDUCACIÓN PÚBLICA (MEP). Programas de Estudio de Matemáticas. I, II Y III Ciclos de la Educación General Básica y Ciclo Diversificado. San José: MEP, 2012.

MINISTERIO DE EDUCACIÓN, CULTURA Y DEPORTE. Real Decreto 1105/2014, de 26 de diciembre, por el que se establece el currículo básico de la Educación Secundaria Obligatoria y del Bachillerato. Madrid: MECD, 2014.

NATIONAL COUNCIL OF TEACHERS OF MATHEMATICS (NCTM). Principles and standards for school mathematics. Reston, VA: NCTM, 2000.

NOELTING, Gerald. The development of proportional reasoning and the ratio concept. Part I. Differentiation of stages. Educational Studies in Mathematics, New York, v. 11, n. 2, p. 217-253, 1980a.

NOELTING, Gerald. The development of proportional reasoning and the ratio concept. Part II. Problem structure at successive stages: problem solving strategies and the mechanism of adaptive restructuring. Educational Studies in Mathematics, New York, v. 11, n. 3, p. 331-363, 1980b.

PIAGET, Jean; INHELDER, Bärbel. La genése de l'idée de hasard chez l'enfant. Paris: Presses Universitaires de France, 1951.

PRATT, Dave. The Co-ordination of Meanings for Randomness. For the Learning of Mathematics, Canada, v. 18, n. 3, p. 2-11, 1998.

PRATT, Dave. Making sense of the total of two dice. Journal for Research in mathematics Education, Reston, v. 31, n. 5, p. 602-625, 2000.

TRURAN, John. Examination of a relationship between children's estimation of probabilities and their understanding of proportion. In: PONTE, João Pedro; MATOS, João Filipe (Ed.). Proceedings of the XVIII PME Conference. Universidad de Lisboa, 1994. P. 337-344.

Luis Armando Hernández Solís es Máster en Didáctica de las Matemática de la Universidad de Granada, España. Desde el 2010 es miembro de la Comisión Central del Proyecto Reforma de la Educación Matemática en Costa Rica, del Ministerio de Educación Pública. También es profesor de la carrera de Enseñanza de la Matemática de la Universidad Estatal a Distancia en Costa Rica desde 2007.

ORCID: https://orcid.org/0000-0003-2956-8102

E-mail:lhernandez@uned.ac.cr

Carmen Batanero es Doctora en Matemáticas por la Universidad de Granada (España). Catedrática jubilada de Didáctica de la Matemática, actualmente profesora colaboradora y profesora del Programa de Doctorado en Educación de la Universidad de Granada. Fue miembro del Comité Ejecutivo de ICMI (International Comision on Mathematical Instruction) y Presidenta de IASE (International Association for Statistical Education).

ORCID: https://orcid.org/0000-0002-4189-7139

E-mail: batanero@ugr.es 
$\underline{\text { Resolución de Tareas Probabilísticas en Contexto Geométrico por Estudiantes... }}$

María Magdalena Gea es Profesora Contratada Doctora en el Departamento de Didáctica de la Matemática de la Universidad de Granada. Doctora en Ciencias de la Educación por la Universidad de Granada (España). Licenciada en Matemáticas, Licenciada en Ciencias y Técnicas Estadísticas, Máster en Estadística Aplicada, Máster en Didáctica de la Matemática y Diploma de estudios avanzados.

ORCID: https://orcid.org/0000-0002-5229-0121

E-mail:mmgea@ugr.es

Rocío Álvarez-Arroyo es Profesora Ayudante Doctora en el Departamento de Didáctica de la Matemática de la Universidad de Granada. Doctora en Ingeniería Civil por la Universidad de Granada (España). Ingeniero Químico, Ingeniero Técnico Industrial, Máster en Investigación y Avances en Microbiología y Máster en Didáctica de la Matemática por la Universidad de Granada (España).

ORCID: https://orcid.org/0000-0002-3201-8542

E-mail: rocioaarroyo@ugr.es

Editora a cargo: Carla Vasques

Este é um artigo de acesso aberto distribuído sob os termos de uma Licença Creative Commons Atribuição 4.0 Internacional. Disponível em: <http:// creativecommons.org/licenses/by/4.0>. 